\title{
Nevralgia do pudendo: um desafio diagnóstico
}

Raquel Lobo Cardoso, ${ }^{1}$ Diana Rodrigues Pacheco ${ }^{1}$

\section{RESUMO}

Introdução: A nevralgia do pudendo define-se como uma dor ao longo do dermátomo do nervo pudendo e pode ocorrer em ambos os sexos. Sendo uma patologia capaz de mimetizar muitas outras condições orgânicas, o seu diagnóstico surge geralmente após meses de sintomatologia. O presente relato de caso pretende enfatizar uma patologia rara, alguns dos seus aspetos clínicos e critérios de diagnóstico, demonstrando um exemplo de como o atraso no diagnóstico pode conduzir a um excessivo gasto de recursos em saúde, bem como a um sofrimento físico e psicológico significativos.

Descrição do caso: Utente do sexo feminino, de 73 anos, recorre à consulta aberta da Unidade de Saúde Familiar (USF), em agosto 2016, por queixas de ardência vulvar e desconforto na região suprapúbica com três semanas de evolução. Realizou antibioterapia empírica, sem melhoria da sintomatologia. Por manutenção das queixas recorre a várias consultas de medicina geral e familiar na USF e a várias especialidades em regime público e privado, sendo medicada com vários antibióticos, antifúngicos, corticoides, entre outros. Foram realizados vários exames auxiliares de diagnóstico e levantadas inúmeras hipóteses diagnósticas até que, mais de um ano desde o surgimento dos primeiros sintomas, se chega ao diagnóstico mais provável de nevralgia do pudendo. Apesar do diagnóstico, a doente continuou com a dor mal controlada, tendo mesmo de recorrer às opções de último recurso para o tratamento desta patologia.

Comentário: A nevralgia do pudendo é uma patologia cujo diagnóstico se alcança habitualmente após muito tempo do início da sintomatologia do doente, com muito sofrimento físico e psicológico associado. Por este motivo, o relato de caso apresentado pretende sensibilizar o profissional de saúde para este diagnóstico e para a importância do médico de família enquanto elemento integrador dos cuidados do doente.

Palavras-chave: Nervo pudendo; Nevralgia; Medicina geral e familiar.

\section{INTRODUÇÃO}

A nevralgia do pudendo é uma condição rara que envolve o nervo pudendo e que pode ocorrer em ambos os sexos..$^{1-3}$ Define-se como uma dor ao longo do dermátomo do nervo pudendo, localizando-se assim na vulva, vagina, clitóris, períneo e reto no sexo feminino e na glande, escroto, períneo e reto no sexo masculino. ${ }^{1-3}$ Pode ser descrita como uma dor de características neuropáticas (em queimadura, facada ou adormecimento). ${ }^{4}$

A etiologia da nevralgia do pudendo nem sempre é clara, mas acredita-se que pode ser provocada pelo estiramento ou compressão nervosa, sendo várias as causas propostas na literatura de lesão do nervo pudendo. ${ }^{1-3}$ Dada a variedade de patologias orgânicas muito mais comuns que podem provocar sintomas nesta região, a

1. Médica Interna de Medicina Geral e Familiar. USF Horizonte, ULS de Matosinhos. Matosinhos, Portugal. nevralgia do pudendo é, muitas vezes, a última opção a ser colocada, sendo geralmente um diagnóstico de exclusão que surge tardiamente na evolução do quadro clínico. $^{3}$

O diagnóstico de nevralgia do pudendo requer um alto grau de suspeição, sendo essencialmente clínico. Apesar de não existirem critérios patognomónicos há várias características que podem sugerir o diagnóstico. ${ }^{4}$

O tratamento desta patologia compreende desde medidas de modificação comportamental (eviç̧ão de fatores desencadeantes da dor), fisioterapia, tratamento farmacológico com gabapentina e antidepressivos tricíclicos, até ao bloqueio do nervo pudendo, descompressão cirúrgica e neuromodulação. ${ }^{3}$

O presente relato de caso pretende relembrar uma patologia que, embora rara, pode causar sofrimento físico e psicológico significativo. Os sintomas manifestados pelo utente podem confundir e mimetizar outras patologias, dificultar o diagnóstico atempado, 
conduzir a um desproporcionado gasto de recursos em saúde e levar à instituição de terapêutica ineficaz e, por vezes, não dirigida ao problema. Pretende-se ainda destacar a apresentação clínica e os critérios de diagnóstico da nevralgia do pudendo.

\section{DESCRIÇÃO DO CASO}

História e antecedentes pessoais

Utente do sexo feminino, de 73 anos, caucasiana, casada, licenciada, atualmente reformada, inserida numa família nuclear em fase VIII do ciclo de vida de Duvall. Tem, como antecedentes pessoais, obstipação crónica [com necessidade de recurso a laxantes, vários dias por semana e descrita como presente "desde sempre" (sic)], melanoma do braço diagnosticado aos 62 anos, insónia inicial e sono descrito como "não repador" desde os 67 anos. Como antecedentes cirúrgicos destaque para apendicectomia aos 18 anos, histerectomia com anexectomia bilateral por hemorragia uterina anómala aos 50 anos, colecistectomia aos 61 anos e excisão de melanoma aos 62 anos. Do foro ginecológico e obstétrico a destacar: IIGIIP, partos distócicos, com necessidade de episiotomia (peso dos recém-nascidos ao nascimento: $3 \mathrm{~kg}$ e $5 \mathrm{~kg}$ ).

\section{História da doença atual}

Em agosto de 2016 a utente recorre à consulta aberta da sua Unidade de Saúde Familiar (USF) com queixas de ardência vulvar (por vezes associada a disúria) e desconforto na região suprapúbica com três semanas de evolução. A utente menciona também que urina com muita frequência, mas que este seria o seu padrão habitual, referindo ainda secura vaginal com anos de evolução. Nega outra sintomatologia, nomeadamente prurido vulvar, leucorreia, urgência urinária, dor lombar ou febre. Nega fator ou evento desencadeante dos sintomas. Até ao momento, a utente já tinha recorrido a duas consultas em regime particular, tendo sido feito o diagnóstico de infeção urinária, não tendo até então realizado nenhum exame complementar de diagnóstico, nomeadamente urocultura. Tinha sido medicada, na primeira consulta, com nitrofurantoína e, na segunda, com amoxicilina com ácido clavulânico, sem resolução das queixas. Como medicação habitual realizava trazodona e laxante à base de extrato de sene. Ao exame físico apresentava atrofia vaginal, sem outras alterações. Foi reali- zado teste com tira teste urinária que revelou apenas presença de leucócitos (125 células/ $\mu \mathrm{L}$ ). Foi feito o aconselhamento e foi medicada com fosfomicina e estriol vaginal. Foram pedidos exame sumário de urina e urocultura para realizar antes da toma do antibiótico, não tendo estes exames revelado alterações.

No início de setembro de 2016, em consulta programada, a utente volta a referir queixas de ardência nos grandes lábios e região anal, que surge ou se intensifica significativamente quando se senta e que vai agravando ao longo do dia. A dor não acorda a utente durante a noite; no entanto, refere ter um sono não reparador. Por outro lado, com o ortostatismo ou quando caminha não sente tanta dor e tem alívio significativo quando se senta durante uns minutos na sanita. A utente refere que não consegue usar roupas justas porque o simples toque da roupa na região vulvar e anal desperta dor. Menciona também dispareunia profunda. A dor não cede com paracetamol ou ibuprofeno. Na escala numérica da dor, a utente classifica a dor como um oito/nove. De destacar também a obstipação, já de longa data, mas sem alteração do padrão. Nega queixas relacionadas com o trato urinário. De ressalvar a interferência marcada da dor na vida diária da utente, que levou mesmo à evicção de situações sociais que implicassem permanência na posição sentada durante longos períodos, como sejam convívios de família ou com amigos, viagens e outras atividades de lazer. Para além dos três antibióticos referidos anteriormente tinha feito itraconazol, com ligeira melhoria. Tinha consulta agendada dentro de 15 dias em ginecologista particular e, por esse motivo, nesta consulta não foi implementada mais nenhuma medida a não ser o aconselhamento quanto a medidas corretoras da obstipação, nomeadamente aumento da ingestão de fibras e hídrica e prática de exercício físico regular, e cuidados gerais da região genital, como higiene íntima, tipo de vestuário e cuidados posturais.

No final de setembro de 2016 recorre a consulta aberta da USF, por manutenção das queixas, descritas nesse momento como "ardência e acidez uretral" (sic). Já tinha tido consulta em ginecologista particular, tendo sido medicada com fluticasona tópica e ciprofloxacina oral, que tinha terminado dois dias antes, sentindo que piorou com o corticoide tópico. Negava leucorreia ou outros sintomas. Ao exame físico apresentava atrofia da 
mucosa vulvovaginal, tendo sido medicada com sulfametoxazol + trimetoprim. No início de outubro de 2016 recorre novamente à consulta aberta da USF, por manutenção das queixas e para mostrar exames que tinha realizado. Estava a ser seguida em urologia, ginecologia e nutrição, em regime privado, que consideraram, entre outras causas, que os sintomas se pudessem dever a algum tipo de alergia alimentar. A utente realizou então dieta com evicção de vários alimentos, sequencialmente, de forma a perceber se se tratava de alguma alergia, não tendo encontrado nenhum alimento causador dos sintomas. Dos exames realizados em regime privado (ecografia renal e vesical, ecografia pélvica, citologia cervico-vaginal, colheita de exsudado vaginal) nenhum demonstrou alterações de relevo. Como a utente já se encontrava a ser seguida em várias especialidades em regime particular optou-se, nesta consulta, por não instituir mais nenhum tratamento, tendo-se agendado consulta programada dentro de 15 dias para reavaliação.

Na consulta de reavaliação, no final de outubro, a utente mantinha a sintomatologia referida anteriormente, encontrando-se apenas medicada com estriol vaginal. Ao exame ginecológico mantinha-se a atrofia vulvovaginal, sem outras alterações. Foi novamente aconselhada quanto às medidas corretoras da obstipação e aos cuidados gerais da região genital, tendo sido prescrito estriol e Lactobacillus acidophilus vaginal.

Em janeiro de 2017, em consulta programada na USF agendada por manutenção das queixas, a utente referiu que já tinha recorrido a quatro ginecologistas diferentes, em regime privado, pelo mesmo motivo. Aguardava também consulta de medicina da dor, em hospital público (referenciação realizada através de médico particular). Não foram efetuadas quaisquer alterações terapêuticas ou intervenções diagnósticas nesta consulta. No entanto, para além dos aconselhamentos já previamente realizados, foi também aconselhada a manter seguimento com o médico assistente, gestor dos cuidados de saúde, que pudesse integrar toda a informação das diversas especialidades e, em conjunto com a utente, estabelecer um plano integrado de cuidados.

Em fevereiro de 2017, a utente teve a primeira consulta de medicina da dor. Foram descritos pontos dolorosos na área dos pequenos e grandes lábios vaginais e sensibilidade aumentada ao nível das espinhas is- quiáticas e ligamentos sacrotuberais bilateralmente. Foram negadas alterações da sensibilidade na região genital ou anal. Quando recorreu a esta consulta a utente tinha já realizado ressonância magnética (RMN) pélvica e biópsia vulvovaginal. A biópsia não revelou nenhuma alteração de relevo. Destacam-se, no entanto, os achados presentes na RMN: "falência dos músculos do pavimento pélvico, sinais de fibrose, cistocelo e retocelo". Tendo em conta a história clínica, antecedentes pessoais (partos traumáticos, cirurgias abdominopélvicas e obstipação crónica), o diagnóstico de nevralgia do pudendo surgiu como hipótese (ainda por confirmar) e a utente foi medicada com gabapentina oral e lidocaína tópica. Foi aconselhada à realização de fisioterapia de reforço do pavimento pélvico.

De março a maio de 2017 a utente teve mais três consultas de medicina da dor, tendo sido aumentada a dose de gabapentina e associada amitriptilina ao tratamento, por dor não controlada. Na consulta de maio foi agendado o procedimento de bloqueio do nervo pudendo, ao qual a doente faltou.

Em junho de 2017 a utente solicita consulta programada com a sua médica de família para, segundo a própria, "fazer um ponto da situação" (sic). Mantinha as mesmas queixas, tendo já tido consulta nas seguintes especialidades em regime privado: ginecologia, urologia, gastroenterologia, imunoalergologia, dermatologia, cirurgia vascular, ortopedia, psiquiatria e nutrição. Estava também a ser seguida em medicina da dor, sem controlo satisfatório da sintomatologia.

Até ao momento tinham sido levantadas várias hipóteses diagnósticas: infeção urinária e/ou genital, cistite crónica, alergia alimentar, doença autoimune, varizes vulvares, nevralgia do pudendo. De ressalvar que, devido à inacessibilidade dos registos efetuados em regime particular, não se conseguiu apurar todas as hipóteses diagnósticas nem perceber por que especialidades foram levantadas, com exceção da hipótese diagnóstica de nevralgia do pudendo, colocada na consulta de medicina da dor, onde a doente era seguida em hospital público.

Em termos terapêuticos, já tinha sido medicada com diversos fármacos orais (claritromicina, metronidazol, fluconazol, aciclovir, linaclotida, diosmina, citrato de potássio, colecalciferol, glicerofosfato de cálcio, ácido fólico, terapêutica hormonal de substituição com estra- 
diol, complexos multivitamínicos, suplemento alimentar com probióticos) e fármacos tópicos (cloreto de dequalínio, metronidazol, ciclopirox, nitrato de econazol, miconazol, hidrocortisona, lidocaína, trolamina, Lactobacillus plantarum, Lactobacillus rhamnosus LB21, vários cremes hidratantes vaginais e vulvares), para além dos já previamente descritos. De notar que não se conseguiu averiguar a ordem pela qual foram prescritos, pela inacessibilidade a alguns registos médicos.

Após mais três consultas de seguimento de medicina da dor e por manutenção de dor não controlada realizou, em dezembro de 2017, bloqueio e radiofrequência pulsada dos nervos pudendos por via anterior com anestesia geral, tendo obtido melhoria significativa da dor. Assim, após cerca de um ano e meio após o início de sintomas e com a melhoria sintomática obtida com o procedimento, foi estabelecido o diagnóstico de nevralgia do pudendo. No entanto, a melhoria obtida após este procedimento durou apenas cerca de um mês, voltando depois a utente a apresentar sintomatologia marcada, sem nenhum evento desencadeante descrito. Apresentava apenas melhorias ligeiras com toda a medicação que lhe tinha sido oferecida, exibindo acentuação de sintomas ansiosos e depressivos reativos ao quadro clínico, que se iniciaram cerca de três meses após o surgimento dos sintomas, sendo por esse motivo orientada para psicologia.

No início de 2018 começou a ser seguida em consulta de cirurgia geral, por iniciativa própria, tendo, em maio, sido submetida a descompressão cirúrgica do nervo pudendo. Esta cirurgia levou a uma melhoria substancial das queixas da utente, que se manteve com um excelente controlo da dor até abril de 2019, altura em que sofreu novo agravamento da sintomatologia.

Nas consultas de medicina da dor foi ponderada a repetição de radiofrequência pulsada do nervo pudendo. No entanto, a utente não se encontrava motivada para a sua realização.

Em outubro de 2019, aconselhada por ginecologista em regime particular, adotou uma série de mudanças comportamentais de controlo da dor (não permanece longos períodos sentada, usa vestuário mais largo, faz caminhadas diárias, aplica frio localmente nos períodos de agravamento) e iniciou novamente fisioterapia dirigida ao pavimento pélvico, com melhoria acentuada das queixas.
Na última consulta realizada na USF, em fevereiro de 2020 , a utente encontrava-se a realizar fisioterapia de reforço do pavimento pélvico e mantinha seguimento em consulta de medicina da dor e ginecologia. Estava medicada com pregabalina, lorazepam, estrogénio e probióticos vaginais e supositório de glicerina. A dor atualmente é descrita como ardência vulvar (mais no grande lábio esquerdo), sem irradiação, que agrava ou surge na posição de sentada e ao longo do dia e alivia com o ortostatismo, com a marcha e com a aplicação de frio. Não voltou a ter queixas na região anal desde a cirurgia. A utente reconhece que, apesar de ter sofrido agravamento da sintomatologia em abril de 2019 (depois da cirurgia de descompressão), não voltou a ter níveis tão intensos nem a mesma frequência de dor que tinha anteriormente $\mathrm{e}$ que atualmente a dor é suportável, está controlada durante a maior parte do dia e que esta não interfere no seu quotidiano. A utente associa o controlo atual da dor principalmente às mudanças comportamentais que adotou $\mathrm{e}$ à fisioterapia que se encontra a realizar.

\section{COMENTÁRIO}

Entre as competências profissionais do médico de família definidas pela WONCA, encontra-se o uso eficiente dos recursos de saúde, coordenando os cuidados e gerindo a interface com outras especialidades, assumindo um papel de advocacia quando necessário. ${ }^{5}$

Essa função de coordenação é uma característica essencial de custo-efetividade nos cuidados de saúde primários, assegurando que os pacientes recorrem ao profissional de saúde mais adequado para o seu problema específico. O médico de família pode desempenhar um papel central, de concentração da informação clínica, resumo e orientação posterior, se indicado. No entanto, nem sempre se reúnem as condições estruturais necessárias para esta prática. Neste caso, a procura, por parte da utente, de diferentes especialidades em diferentes unidades de saúde, públicas e privadas, condicionou este papel de concentração de informação, dificultando o papel do médico de família de "gestor dos problemas de saúde" da utente e poderá ter, em parte, dificultado o diagnóstico mais precoce da sua situação clínica. Apesar de a utente recorrer várias vezes ao centro de saúde, vários destes recursos foram na consulta aberta da unidade, onde foi observada por diferentes médicos. Em todas as consultas programadas, a 
utente referia sempre as queixas, mas apresentava-as como já orientadas por outros médicos, sendo difícil demover a utente do recurso a todas as especialidades a que recorria e fazer uma orientação precisa do seu caso.

A fácil acessibilidade a várias especialidades médicas, em regime particular, aliada à falta de comunicação entre os vários médicos e à não acessibilidade dos médicos do regime público à informação clínica registada em regime particular (e vice-versa) levaram a que fossem colocadas várias hipóteses diagnósticas (duplicadas entre os vários médicos), sendo a utente submetida a vários tratamentos, muitas vezes da mesma classe terapêutica, sem melhoria dos sintomas e com sofrimento físico e psicológico significativo e evitáveis.

Outro aspeto a ressalvar é a iatrogenia presente neste caso. Começa desde logo nos antecedentes da doente: a obstipação crónica com recurso a laxantes várias vezes por semana (não se sabendo se foram otimizadas ao máximo as medidas de estilo de vida previamente à introdução dos laxantes) e as cirurgias a que foi submetida. Durante a evolução deste quadro clínico é também evidente a iatrogenia nos vários exames complementares de diagnóstico que realizou e nos vários tratamentos a que foi submetida.

Para além do já referido, a procura incessante por um diagnóstico e tratamento eficaz acarretou também um excessivo gasto de recursos em saúde. Um uso mais adequado destes recursos poderia ter sido alcançado se tivesse havido comunicação entre os vários médicos e se tivesse sido facilitado ao médico de família exercer a sua função de «orientador dos cuidados».

Na verdade, perante queixas de dor na região pélvica, a nevralgia do nervo pudendo é muitas vezes um diagnóstico de exclusão, que surge tardiamente na evolução do quadro clínico, exigindo um elevado nível de suspeição. $\cdot^{3-4}$ Apesar de não existirem critérios patognomónicos, existem alguns sugestivos do diagnóstico. ${ }^{3-4}$

Efetivamente, são cinco os critérios considerados essenciais que devem estar presentes em simultâneo para se chegar ao diagnóstico de nevralgia do pudendo e que se apresentam: (1) dor no território do nervo pudendo, do ânus até ao pénis ou clitóris; (2) a dor é predominantemente sentida quando o utente está sentado, sendo que a dor é devida à excessiva pressão sobre o nervo e não à posição de sentar, como é ilustrado pelo alívio da dor quando o utente se senta na sanita; (3) a dor não acorda o paciente à noite (apenas excecionalmente é reportada história de despertar noturno pela dor, mas estes episódios são raros); (4) dor sem diminuição objetiva da sensibilidade; (5) a dor é aliviada por bloqueio do nervo pudendo. ${ }^{4}$

Para além de estarem presentes os cincos critérios acima mencionados, o caso apresentado exibe também alguns critérios complementares, nomeadamente dor de características neuropáticas (descrita como ardência/queimadura); alodínia (dor com o simples toque da roupa); hiperalgesia e sensibilidade aumentada à palpação da espinha isquiática (descritos no exame físico na consulta de medicina da dor) e agravamento da dor durante o dia. ${ }^{4}$

A existência de partos traumáticos, de cirurgias abdomino-pélvicas prévias e de obstipação crónica, com consequente lesão por estiramento, compressão ou mesmo transecção do nervo, são possíveis causas de nevralgia do pudendo neste caso clínico. ${ }^{1-2}$

Apesar de retrospetivamente este diagnóstico parecer simples, tendo em conta que estavam presentes quatro dos cinco critérios essenciais para o diagnóstico (tendo sido completado o quinto critério após a realização do bloqueio do nervo pudendo, com alívio da sintomatologia) e ainda vários complementares, é de ressalvar que outras patologias se podem enquadrar nestes critérios e que, portanto, várias doenças orgânicas devem ser excluídas antes de se estabelecer o diagnóstico de nevralgia do pudendo. Este aspeto contribui para a dificuldade e atraso no diagnóstico e tratamento atempado desta patologia.

Em suma, o presente caso demonstra a necessidade de sensibilizar o médico de família para a nevralgia do pudendo e realçar as características clínicas que, apesar de não serem patognomónicas, poderão auxiliar no seu diagnóstico. Para além disso, este caso é também uma demonstração do quão importante é o médico de família na gestão dos problemas do utente, evitando que este se sinta assoberbado por várias opiniões clínicas divergentes, contribuindo, assim, para uma orientação mais eficaz, com menos gastos em saúde e menos sofrimento físico e psicológico para o utente.

\section{REFERÊNCIAS BIBLIOGRÁFICAS}

1. Benson JT, Griffis K. Pudendal neuralgia, a severe pain syndrome. Am J Obstet Gynecol. 2005;192(5):1663-8. 
2. Khoder W, Hale D. Pudendal neuralgia. Obstet Gynecol Clin North Am. 2014;41(3):443-52.

3. Stav K, Dwyer P, Roberts L. Pudendal neuralgia: fact or fiction? Obstet Gynecol Surv. 2009;64(3):190-9.

4. Labat JJ, Riant T, Robert R, Amarenco G, Lefaucheur JP, Rigaud J. Diagnostic criteria for pudendal neuralgia by pudendal nerve entrapment (Nantes criteria). Neurourol Urodyn. 2008;27(4):306-10.

5. Allen J, Gay B, Crebolder H, Heyrman J, Svab I, Ram P. The European definition of GP/FM [homepage].WONCA Europe; 2011. Available from: https://www.woncaeurope.org/page/definition-of-general-practice-family-medicine

\section{CONSENTIMENTO INFORMADO}

A utente deu o seu consentimento informado, livre e esclarecido para a recolha de dados clínicos, assim como para publicação.

\section{CONFLITO DE INTERESSES}

As autoras declaram não possuir quaisquer conflitos de interesse.

\section{FINANCIAMENTO DO TRABALHO}

As autoras declaram que o trabalho relatado neste manuscrito não foi objeto de qualquer tipo de financiamento externo.

\section{ENDEREÇO PARA CORRESPONDÊNCIA}

Raquel Lobo Cardoso

E-mail: raquellobocardoso@gmail.com

https://orcid.org/0000-0003-0841-2555

Recebido em 30-05-2020

Aceite para publicação em 18-12-2020

\section{ABSTRACT}

\section{PUDENDAL NEURALGIA: A DIAGNOSTIC CHALLENGE}

Introduction: Pudendal neuralgia is defined as pain along the dermatome of the pudendal nerve and can occur in both sexes. Being a pathology capable of mimicking many other organic conditions, its diagnosis usually appears after months of symptoms. The present case report aims to emphasize a rare pathology, some of its clinical aspects, and diagnostic criteria, demonstrating an example of how the delay in the diagnosis can lead to excessive spending of health resources as well as to significant physical and psychological suffering.

Case description: A 73-year-old female patient resorts to a medical appointment in the Family Health Unit in August 2016 for complaints of vulvar burning and discomfort in the suprapubic region with three weeks of evolution. Empirical antibiotic therapy was performed, with no improvement in symptoms. Because of the complaints' persistence, she resorts to several appointments at the Family Health Unit and various medical specialties in the public and private regime, being medicated with various antibiotics, antifungals, corticosteroids, among others. Several auxiliary diagnostic tests have been carried out and numerous diagnostic hypotheses have been raised until more than a year since the appearance of the first symptoms, the most probable diagnosis of pudendal neuralgia is established. Despite the diagnosis, the patient continued to have poorly controlled pain, having to resort to options of the last resource for the treatment of this pathology.

Comments: Pudendal neuralgia is a condition whose diagnosis is usually reached long after the patient's symptoms have started, with a lot of associated physical and psychological suffering. For this reason, the case report presented pretends to sensitize the health professional to this diagnosis and to the importance of the family doctor as an integrating element of the patient's care.

Keywords: Pudendal nerve; Neuralgia; Family practice. 